PROC. OF JSCE,

No. 230, OCT. 1974

\title{
AN ANALYTICAL STUDY OF TWO-LAYERED, RAPIDLY VARIED FLOW
}

\author{
By Norio HAYAKAWA*
}

\section{INTRODUCTION}

Parallel streams of two-layered fluids exhibit various flow characteristics and are frequently observed in an aquatic environment. Understanding of this kind of phenomenon finds increasing applications in present-day world of industrialization and endangered eco-system.

Two-layered parallel streams may be or may not be accompanied by strong mixing. Specifically, the flow will resemble any of jet, hydraulic jump or immiscible two-layered fluids. Classification of these flow regimes and their possible occurrence are discussed by, among others, $\mathrm{Koh}^{1)}$ and Stefan ${ }^{2}$.

Two-layered rapidly varied flow encompasses jet and hydraulic jump. Characteristic to this class of flow is that the flow is internally supercritical and very often accompanied by strong mixing. On account of this mixing, the flow loses its momentum and often is followed by a two-layered flow system which is internally subcritical and accompanied by a neglible amount of mixing. Transition from supercritical to subcritical regime is tractable by the impulsemomentum principle.

Such an analysis was first presented by Schijf and Schönfeld ${ }^{3)}$ who solved a case when one fluid layer is in motion and there is no mixing between the layers. More general treatment to the case when two layers are in co-current motion is given by Yih and Guhat). Shi-ighai ${ }^{5)}$, Hamada ${ }^{6}$, and Iwasaki ${ }^{7}$ ) studied the solution of the momentum equations under the premise that the free water surface remains level. Among them, Iwasaki gave energy relationship of internal hydraulic jump. On the basis of this Hayakawa ${ }^{8)}$ refined analysis given by Yih and Guha.

All the works mentioned above relate to internal

\footnotetext{
* Chief Research Engineer, Chugoku Institute of Industrial Technology, MITI, 15000 Hiromachi, Kure.
}

hydraulic jump of immiscible fluids. Mixing and turbulence associated with internal hydraulic jump has been studied only recently. Thus Iwasaki and Uehara ${ }^{9)}$, and Iwasaki and $\mathrm{Abe}^{10)}$ reported their extensive study on turbulent structure related to internal hydraulic jump. Wood ${ }^{11)}$ has reported his experiments and some analysis on mixing internal hydraulic jump. Wilkinson and Wood $^{12)}$ reported observation of various flow regimes with wide range of mixing when the lower layer was quiescent and the upper layer was covered by the solid wall. Similar discussion was presented by Stefan $^{2)}$ regarding with the case the upper layer possesses free surface and an analysis of internal hydraulic jump with mixing considered and a lower layer quiescent was presented by Stefan and Hayakawa ${ }^{13)}$ who solved the impulse-momentum equation for the entire fluid surrounding jump phenomenon assuming hydrostatic pressure distribution in the lower layer across the control volume. Hayakawa and Stefan $^{14)}$ slightly improved their earlier analysis deriving the impulse-momentum equations for both layers.

The present work tries to modify and expand the work of Hayakawa and Stefan. It is first asserted that the rapidly varied flow phenomenon generally consists of jet-type flow followed by internal hydraulic jump (Koh' ${ }^{1)}$ and Stefan $\left.{ }^{2)}\right)$. Therefore, the impulse-momentum equation is applicable to this system and gives the conjugate depth relationship with the rate of mixing as a parameter or vice versa. This is also a repetition of an assertion of Stefan and Hayakawa ${ }^{13)}$ that use of the impulse-momentum equation alone, i.e. without detailed knowledge of the flow inside of the control volume, does not yield a complete solution to the problem with interfacial mixing taken into consideration.

\section{DERIVATION OF GOVERNING EQUATIONS}

The problem treated in this work is shown dia- 


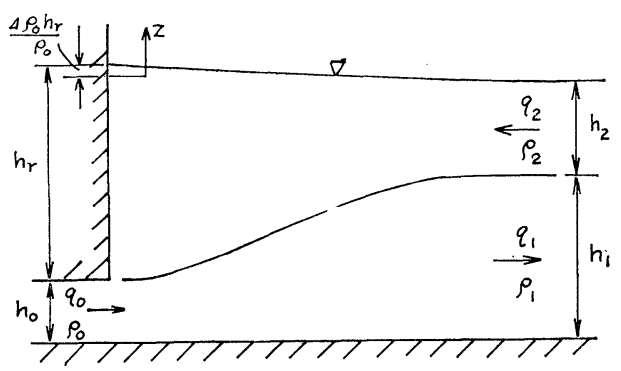

Fig. 1 (a) Definition Sketch of Subsurface Flow.

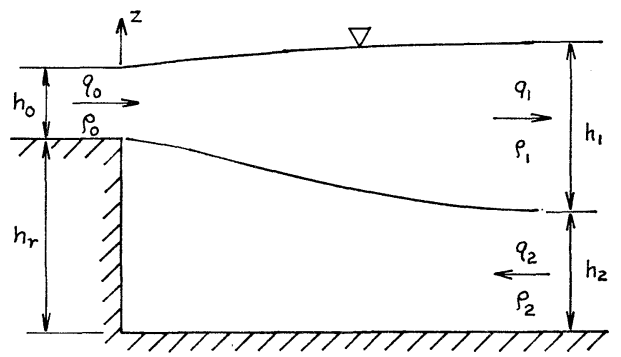

Fig. 1 (b) Definition Sketch of Surface Flow.

grammatically in Fig. 1(a) and Fig. 1(b). In Fig. 1 (a) heavier fluid is discharged along the botton surface of the reservoir (subsurface flow), whereas in Fig. 1(b) lighter fluid is discharged onto the surface of the reservoir (surface flow). The problem is two-dimensional and any interaction with atmosphere such as heat transport is not considered. Fluid is treated essentially as incompressible and miscible. To derive the impulse momentum equation for each layer, it is assumed that the interface is fairly well definable. Furthermore, following three important premises are elected: (I) Fluid being entrained from one layer to another carries a negligible amount of momentum in the longitudinal direction, (II) The pressure distribution at the upstream end of the reservoir is approximated by the hydrostatic distribution, and (III) The average pressure along the interface of the two fluids is the arithematic average value of pressures at the upstream and downstream ends of the control volume. The premise (III) follows Yih and Guhat) whose result is reproduced in the present work when the mixing is assumed to be negligible. Other two premises (I) and (II) exactly hold when mixing is of negligible amount. It should be regarded, therefore, that the present analysis is a good approximation when entrainment is small.

With this preparation the impulse-momentum equations for Fig. 1(a) are written as follows:

$$
\begin{aligned}
\beta_{1} \frac{\rho_{1} q_{1}^{2}}{h_{1}} & -\beta_{0} \frac{\rho_{0} q_{0}^{2}}{h_{0}} \\
= & \frac{1}{2} \rho_{0} g h_{0}^{2}+\rho_{2} g h_{r} h_{0}-\frac{1}{2} \rho_{1} g h_{1}^{2} \\
& -\rho_{2} g h_{1} h_{2}+\frac{\left(h_{1}-h_{0}\right)}{2}\left(\rho_{2} g h_{r}+\rho_{2} g h_{2}\right)
\end{aligned}
$$$$
\text { for lower layer }
$$

$$
\begin{aligned}
\beta_{2} \frac{\rho_{2} q_{2}^{2}}{h_{2}}= & \frac{1}{2} \rho_{2} g h_{r}^{2}-\frac{1}{2} \rho_{2} g h_{2}{ }^{2} \\
& -\frac{\left(h_{1}-h_{0}\right)}{2}\left(\rho_{2} g h_{r}+\rho_{2} g h_{2}\right)
\end{aligned}
$$

for upper layer

where $\beta$ is the momentum correction factor, $\rho$ is fluid density, $q$ is volumetric flow rate per unit width, $h$ is fluid depth and $g$ is the gravitational acceleration. In equations (1) and (2) suffixes 0 and 1 relate to quantities of the lower layer at the upstream and downstream end respectively and 2 and $r$ those of the upper layer at the downstream and the upstream end respectively. Fluid densities are averaged in each layer.

Similarly the impulse-momentum equations for Fig. $1(\mathrm{~b})$ are written as follows;

$$
\begin{aligned}
& \beta_{1} \frac{\rho_{1} q_{1}^{2}}{h_{1}}-\beta_{0} \frac{\rho_{0} q_{0}{ }^{2}}{h_{0}}=\frac{1}{2} \rho_{0} g h_{0}^{2}-\frac{1}{2} \rho_{1} g h_{1}{ }^{2} \\
& +\frac{\left(h_{r}-h_{2}\right)}{2}\left(\rho_{0} g h_{0}+\rho_{1} g h_{1}\right) \\
& \text { for upper layer } \\
& \beta_{2} \frac{\rho_{2} q_{2}^{2}}{h_{2}}=\rho_{0} g h_{0} h_{r}+\frac{1}{2} \rho_{2} g h_{r}^{2}-\rho_{1} g h_{1} h_{2} \\
& -\frac{1}{2} \rho_{2} g h_{2}^{2}-\frac{\left(h_{r}-h_{2}\right)}{2}\left(\rho_{0} g h_{0}+\rho_{1} g h_{1}\right)
\end{aligned}
$$

for lower layer

where suffixes 0 and 1 relate to quantities of the upper layer at the upstream and downstream end respectively and 2 and $r$ those of the lower layer at the downstream and upstream end respectively.

Two problems will be shown to be expressed in a single set of equations. Addition of equations (1) and (2) and the same procedure for equations (3) and (4) are combined as follows:

$$
\begin{aligned}
\beta_{1} \frac{\rho_{1} q_{1}^{2}}{h_{1}} & -\beta_{0} \frac{\rho_{0} q_{0}^{2}}{h_{0}}+\beta_{2} \frac{\rho_{2} q_{2}^{2}}{h_{2}} \\
= & \frac{1}{2} \rho_{0} g h_{0}^{2}-\frac{1}{2} \rho_{1} g h_{1}^{2}+\frac{1}{2} \rho_{2} g h_{r}{ }^{2} \\
& -\frac{1}{2} \rho_{2} g h_{2}^{2}+\left(\begin{array}{c}
\rho_{2} \\
\rho_{0}
\end{array}\right) g h_{r} h_{0}-\left(\begin{array}{c}
\rho_{2} \\
\rho_{1}
\end{array}\right) g h_{1} h_{2}
\end{aligned}
$$

where the upper and lower values relate to the subsurface and surface flow respectively. Similarly, 
Eqs. (2) and (4) are written in a single form as follows:

$$
\begin{aligned}
2 \beta_{2} \frac{\rho_{2} q_{2}^{2}}{g h_{2}\left(h_{2}+h_{r}\right)}= & \left(\begin{array}{c}
\rho_{2} \\
\rho_{0}
\end{array}\right) h_{0}-\left(\begin{array}{c}
\rho_{2} \\
\rho_{1}
\end{array}\right) h_{1} \\
& +\rho_{2}\left(h_{r}-h_{2}\right) \ldots
\end{aligned}
$$

Equations of continuity and incompressibility are the same for two problems and are written as follows:

$$
\begin{aligned}
& \rho_{0} q_{0}+\rho_{2} q_{2}=\rho_{1} q_{1} \\
& q_{0}+q_{2}=q_{1} \quad \cdots \ldots . .
\end{aligned}
$$

Density excesses at the upstream end $\Delta \rho_{0}$ and at the downstream end $\Delta \rho_{1}$ are defined as follows:

$$
\begin{aligned}
& \Delta \rho_{0}= \pm\left(\rho_{0}-\rho_{2}\right) \\
& \Delta \rho_{1}= \pm\left(\rho_{1}-\rho_{2}\right)
\end{aligned}
$$

where the upper and lower signs relate to subsurface and surface flow respectively. Continuity equation (7) is rewritten using Eqs. (8), (9) and (10) as follows:

$$
\Delta \rho_{0} q_{0}=\Delta \rho_{1} q_{1}
$$

Eliminating $q_{2}$ and $\Delta \rho_{1}$ in Eqs. (5) and (6) using Eqs. (8) and (11) and then combining and expressing them in terms of dimensionless quantities lead to the following:

$$
\begin{aligned}
2 F_{0}^{2}= & H_{2}\left[\left(1-\left(\begin{array}{l}
0 \\
r
\end{array}\right)\right)\left(Q-\left(\begin{array}{l}
0 \\
r
\end{array}\right)\right)-\left(H_{d}-\left(\begin{array}{l}
0 \\
r
\end{array}\right)\right)^{2}\right. \\
& +2 \varphi\left\{H_{2}\left(Q-\left(\begin{array}{l}
0 \\
r
\end{array}\right)\right)+\left(Q+\left(\begin{array}{l}
r \\
0
\end{array}\right)\right)\left(H_{d}\right.\right. \\
& \left.\left.\left.-\left(\begin{array}{l}
0 \\
r
\end{array}\right)\right)\right\}-r\left(Q+\left(\begin{array}{l}
r \\
0
\end{array}\right)\right) \varphi^{2}\right](Q \\
& \left.-\left(\begin{array}{l}
0 \\
r
\end{array}\right)\right)^{-1}\left[\beta_{2}(Q-1)^{2}\right. \\
& +\frac{\beta_{1} H_{2}\left(Q-\left(\begin{array}{l}
0 \\
r
\end{array}\right)\right)\left(Q+\left(\begin{array}{c}
r \\
-r
\end{array}\right)\right)}{H_{d}-r \varphi-\left(\begin{array}{l}
0 \\
r
\end{array}\right)} \\
& \left.-\beta_{0}\left(1+\left(\begin{array}{c}
r \\
-r
\end{array}\right)\right) H_{2}\right]^{-1} \ldots \ldots \ldots \ldots(12)
\end{aligned}
$$

Dimensionless quantities in Eq. (12) are defined as follows:

$$
\begin{aligned}
& Q=\frac{q_{1}}{q_{0}}, \quad H_{1}=\frac{h_{1}}{h_{0}}, \quad H_{2}=\frac{h_{2}}{h_{0}}, \quad H_{r}=\frac{h_{r}}{h_{0}}, \\
& r=\frac{\Delta \rho_{0}}{\rho_{2}}, \quad F_{0}=\frac{q_{0}}{\sqrt{r g h_{0}^{3}}}, \quad H_{d}=1+H_{r}-H_{2}, \\
& \varphi=2 \beta_{2} \frac{F_{0}^{2}(Q-1)^{2}}{H_{2}\left(H_{2}+H_{r}\right)} \cdots \cdots \ldots \ldots \ldots \ldots \ldots \ldots(13)
\end{aligned}
$$

In Eq. (12), the densimetric Froude number $F_{0}$ and the depth ratio $H_{2}$ are the primary independent variables. The flow ratio $Q$ and the depth ratio $H_{d}$ are the primary dependent vari- ables. Eq. (12) cannot give a complete answer, say $q_{1}$ and $h_{1}$ to a given problem, say $q_{0}, h_{0}, h_{r}$ and $h_{2}$. With given dimensionless parameters, $F_{0}, H_{r}$ and $H_{2}$, Eq. (12) gives the flow ratio $Q$ as a function of the depth ratio $H_{d}$ or vice versa. The complete answer to the physical problem cannot be given by the use of the impulsemomentum equations alone.

Change in water surface level is given as follows:

$$
W \equiv \frac{H_{1}+H_{2}-1-H_{r}}{r}=\left(\frac{H_{1}}{Q}-1\right)\left(\begin{array}{l}
0 \\
1
\end{array}\right)-\varphi \cdots
$$

Eq. (14) indicates that the change in water surface level is of the order of $r$. It is to be noted that Eq. (14) with $Q=1$ for surface flow was first given by Shi-ighai ${ }^{5)}$. Eqs. (12) and (14) for surface flow were first obtained by Hayakawa and Stefan ${ }^{14}$.

Energy loss of this flow system may be given by calculation of energy flux as follows:

$$
\Delta E=\frac{q_{0} E_{0}+q_{2} E_{2}-q_{1} E_{1}}{q_{0} E_{0}}
$$

where $E_{0}, E_{1}$ and $E_{2}$ are total pressures of each layer. The reference datum planes to calculate the total pressures are selected so that the resultant equations exhibit the maximum symmetry. They are at the free surface of the upstream discharge for the surface flow (Fig. $1(\mathrm{~b})$ ) and at the depth $\Delta \rho_{0} h_{r} / \rho_{0}$ of the upstream end for the subsurface flow (Fig. 1(a)). With this choice Eq. (15) is calculated as follows:

$$
\begin{aligned}
\Delta E=1 & +\frac{\alpha_{2}}{\alpha_{0}} \frac{(Q-1)^{3}}{\left(1+\left(\begin{array}{c}
r \\
-r
\end{array}\right)\right) H_{2}{ }^{2}} \\
& -\frac{\alpha_{1}}{\alpha_{0}} \frac{\left(Q+\left(\begin{array}{c}
r \\
-r
\end{array}\right)\right) Q^{2}}{\left(1+\left(\begin{array}{c}
r \\
-r
\end{array}\right)\right) H_{1}{ }^{2}} \\
& -\frac{2 W}{\alpha_{0} F_{0}{ }^{2}} \frac{1-\frac{r}{Q}\left(\begin{array}{l}
0 \\
1
\end{array}\right)}{1-\left(\begin{array}{l}
0 \\
r
\end{array}\right)} \\
& -\frac{2}{\alpha_{0} F_{0}{ }^{2}} \frac{1}{1+\left(\begin{array}{c}
r \\
-r
\end{array}\right)}\left(\begin{array}{c}
H_{r}-H_{2} \\
\left(1-\frac{1}{Q}\right) H_{d}
\end{array}\right)
\end{aligned}
$$

Hereafter the parameter $r$ is considered to be very small. This is the case for engineering application of this problem to temperature- or salinity-stratified flow. Therefore, the terms involving $r$ in Eqs. (12) and (16) are neglected in the ensuing analysis. Glance on these equations indicates that Eq. (12) is reduced to a single 
dimensionless equation when $r=0$ whereas Eqs. (14) and (16) give slightly different forms for subsurface and surface flow. Change in water surface level given in Eq. (14) is of the order of $r$ or higher. Therefore, substitution of quantities which satisfy Eq. (12) into Eq. (14) yields this value in the lowest order of $r$.

\section{THEORETICAL ANALYSIS FOR $r=0$}

Eq. (12) for $r=0$ can be further rearranged as follows to enable investigation of its analytical behavior:

$$
\phi(Q) \equiv-2 F_{0}{ }^{2} \frac{\left(H_{a}+H_{2}\right)\left(H_{a}-2 H_{2}+1\right)}{H_{d}\left(H_{d}+2 H_{2}-1\right)} Q^{3}
$$

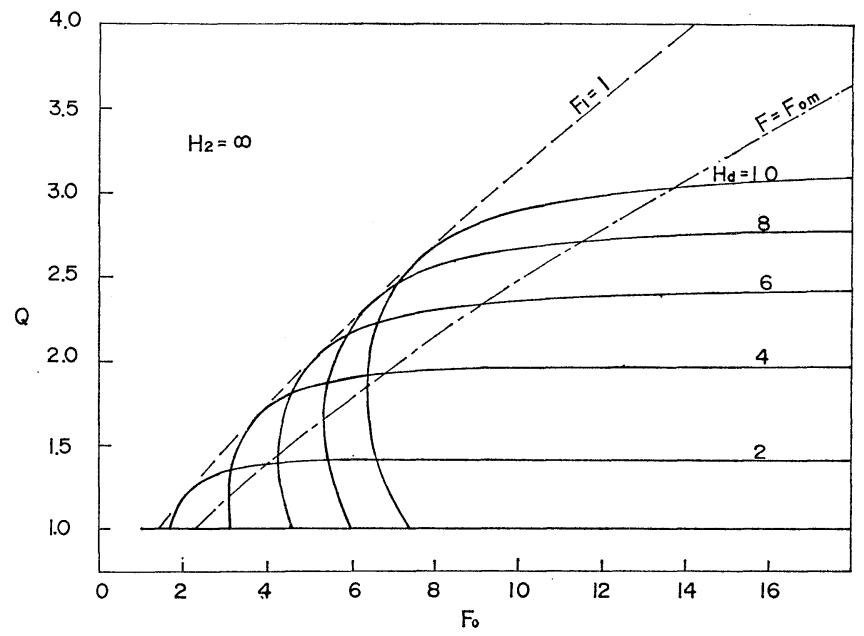

Fig. 2 (a) Theoretical Results for $Q$ as a function of $F_{0}$, $\mathrm{H}_{2} \rightarrow \infty$.

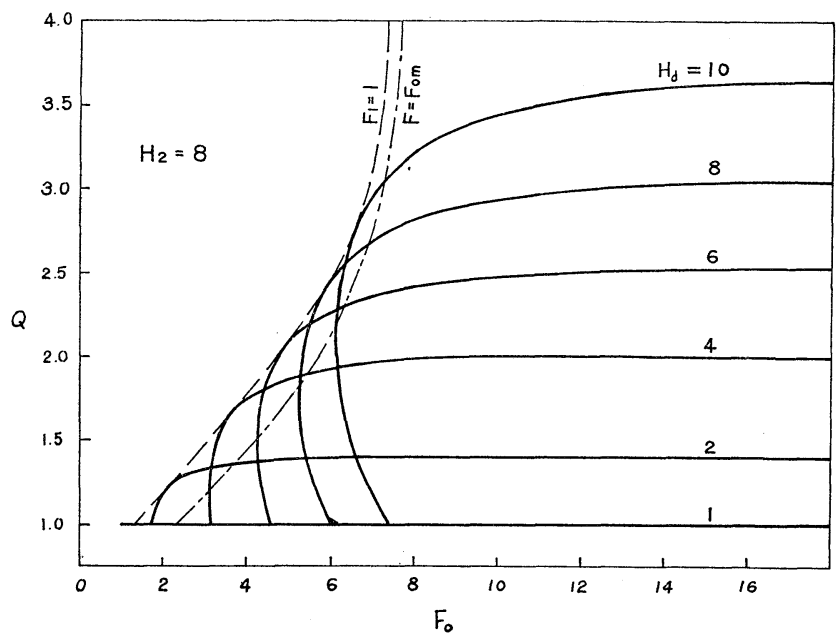

Fig. 2(b) Theoretical Results for $Q$ as a function of $F_{0}$, $H_{2}=8$.

$$
\begin{aligned}
& +4 F_{0}^{2} \frac{H_{d}+1}{H_{d}+2 H_{2}-1} Q^{2} \\
& -2 F_{0}^{2}\left(\frac{H_{d}+1}{H_{d}+2 H_{2}-1}+H_{2}+\frac{H_{2}}{2 F_{0}^{2}}\right) Q \\
& +H_{2} H_{d^{2}}=0
\end{aligned}
$$

If $Q=1$-i.e. with no entrainment-Eq. (17) is solved with respect to $H_{d}$ as follows:

$$
H_{d}=1
$$

or

$$
H_{d}=\frac{1}{2}\left(-1+\sqrt{1+8 F_{0}^{2}}\right)
$$

Equation (18b) is identical to the solution for the non-mixing internal hydraulic jump given by $\mathrm{Yih}$ and Guha ${ }^{4}$. When $Q>1$, Eq. (17) is a cubic equation with respect to $Q$ and can be easily verified that if $H_{a}<2 H_{2}+1$, Eq. (17) has two roots larger than one for $Q$ when $F_{0 m}<F_{0}<F_{\infty}$, where $F_{\infty}$ is given by the inverse relation of Eq. (18b) as a function of $H_{d}$ and $F_{0 m}$ is a number greater than one, and one root when $F_{0}>F_{\infty}$. This point is illustrated in Figs. 2 (a) and 2 (b) which give numerical results of $Q-F_{0}$ relationship for $H_{2}=\infty$ and $H_{2}=8$ respectively. For a given $H_{d}$ the value of $Q$ approaches a constant as $F_{0}$ increases infinitely. The curves of Eq. (17) are bounded by envelopes represented by broken lines labelled as $F_{1}=1$ in Figs. $2(\mathrm{a})$ and $2(\mathrm{~b}) . \quad F_{1}$ is the downstream densimetric Froude number, defined as

$$
\begin{aligned}
F_{1}= & F_{0}\left(\frac{Q^{3}}{H_{d}^{3}}\right)^{1 / 2}\left(1+2 \frac{(Q-1)^{2}}{Q^{2}}\right. \\
& \left.\times \frac{\left(H_{2}-1\right) H_{d}^{2}}{H_{2}\left(H_{d}+2 H_{2}-1\right)}\right) \cdots(19)
\end{aligned}
$$

Each curve for $H_{d}=$ constant in Figs. 2 (a) and 2 (b) has two segments relative to the envelope $F_{1}=1$, one below the point tangent to the envelope and the other above. Only the segment below refers to an internally subcritical flow downstream from the mixing zone, which is necessary to produce an internal hydraulic jump. Thus only the lower segments of the curves $H_{d}=$ constant in Figs. 2 (a) and 2 (b) are physically meaningful as solutions of the internal jump. In Figs. $3(\mathrm{a})$ and $3(\mathrm{~b})$, which give $H_{d}$ $-F_{0}$ relationship, this implies that in each curve for $Q=$ constant the seg- 


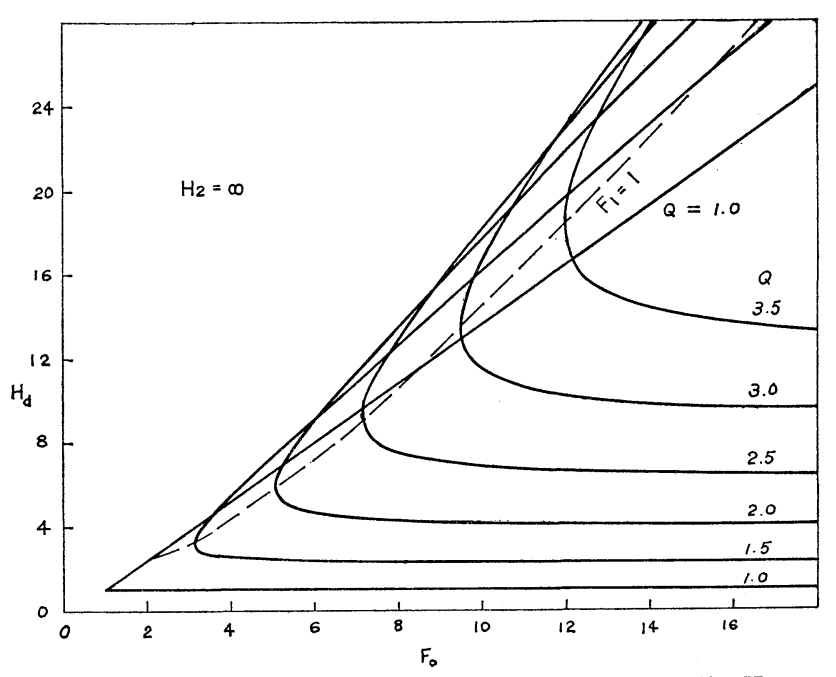

Fig. 3 (a) Theoretical Results for $H_{d}$ as a function of $F_{0}, H_{2} \rightarrow \infty$.

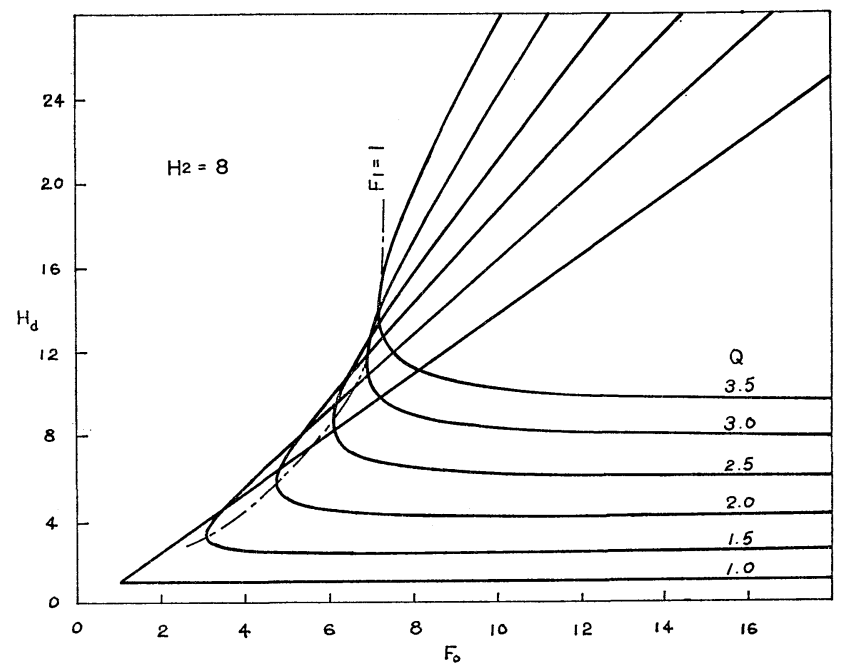

Fig. 3 (b) Theoretical Results for $H_{d}$ as a function of $F_{0}, H_{2}=8$. flow ratio $Q$, respectively, as functions of the densimetric Froude number $F_{0}$. Since the total depth of the experimental tank was limited, the relative thickness of the denser (colder) layer $\mathrm{H}_{2}$ varied within the range $3.5<\mathrm{H}_{2}$ $<13.5$. Fig. 4 indicates that due to the entrainment the downstream depth $H_{d}$ is larger than the depth for a nonmixing internal hydraulic jamp. Furthermore, a majority of the data points fall below the curve of the maximum oberved flow ratio $Q=1.6$ with the average value of the cold layer $H_{2}=8$. Fig. 4 includes a number of data which relate to submerged internal hydraulic jumps. (For description of submerged internal hydraulic jump see Stefan and Hayakawa ${ }^{13)}$.) The submerged internal hydraulic jump involves an additional variable, namely the depth of submergence, and therefore is not tractable in the present analysis. Fig. 4 indicates that the submerged internal hydraulic jump is usually observed in the range $F_{0}<4$ and is associated with greater downstream depths than are predicted by the theory.

Fig. 5 shows a theoretical prediction of the flow ratio, Eq. (17), with $H_{2}=8$, which fits the experimental data remarkably well. In Fig. 5 the theoretical curves, except for the smallest $H_{d}$ value, belong to the portion lower than $F_{0 m}$ line in Fig. 2. Therefore, for a specific set of values for $F_{0}$ and $H_{d}$ (see Fig. 3), the smaller of the two ment above the point of intersection with $F_{1}=1$ is physically meaningful.

Figs. $3(\mathrm{a})$ and $3(\mathrm{~b})$ further illustrate that a point $\left(F_{0}, H_{d}\right)$ above the line $F_{1}=1$ is generally associated with two different $Q$ values which gives the subcritical downstream condition. The experimental data suggest that the smaller value of $Q$ occurs. This point will be discussed further in the next section.

\section{COMPARISON WITH EXPERIMENTAL DATA}

Only Stefan and Hayakawa ${ }^{13)}{ }^{14)}$ reported the data of the entrainment rate of this type of flow. Figures 4 and 5 show the depth ratio $H_{d}$ and the

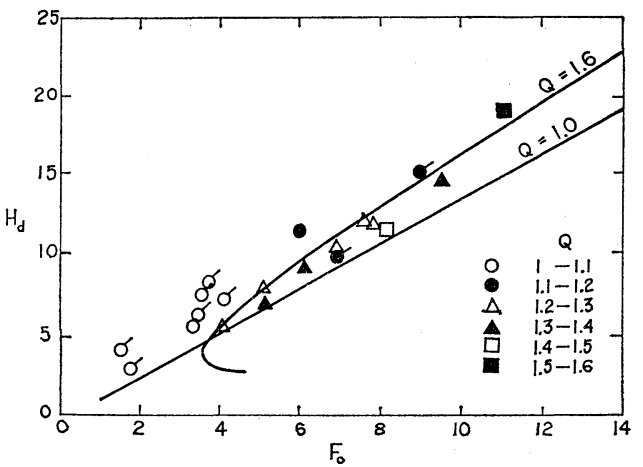

Fig. 4 Comparison with Experimental Data. $H_{d}$ versus $F_{0}$. Solid lines represent Eq. (17) with $H_{2}=8$. Flagged symbols relate to submerged hydraulic jamp. 


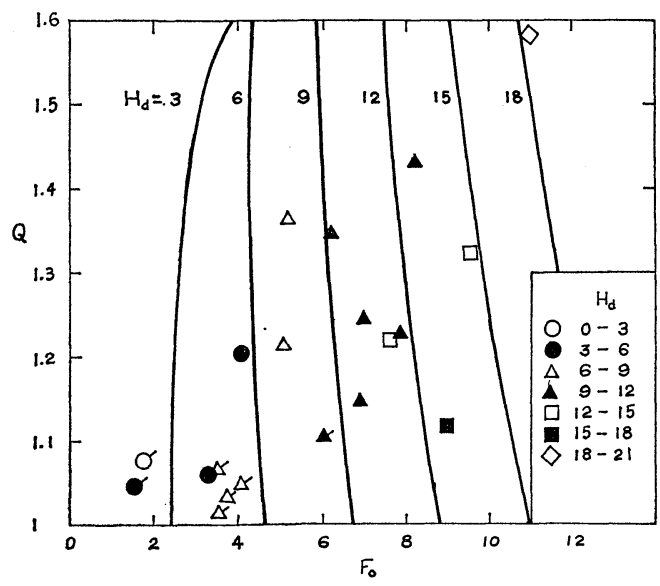

Fig. 5 Comparison with Experimental Data. $Q$ versus $F_{0}$. Solid lines represent Eq. (17) with $H_{2}=8$. Flagged symbols relate to submerged hydraulic jump.

values of $Q$ provided by the theory fits the experimental data. This implies that in Fig. 2 only those segments of the curves which sprout from the $Q=1$ line and terminate at $F_{0}=F_{0 m}$ are physically meaningful.

The flow ratio $Q$ of the experiments never exceeded 1.6, a value which is considerably less than either non-buoyant jets or three-dimensional buoyant surface jets would have produced under similar outfall conditions. The former can be obtained as a limiting value of $Q$ at $F_{0}=\infty$ in Fig. 5, and the latter is reported as high as 10 or more by Harlemen and Stolzenbach ${ }^{15)}$. Fig. 5 further indicates that for a given value of $H_{d}$, an increase in $F_{0}$ results in a sharp decrease in $Q$ except for cases of small $H_{d}$ values. For a given value of $F_{0}$, an increase in $H_{d}$ results in an increase in $Q$.

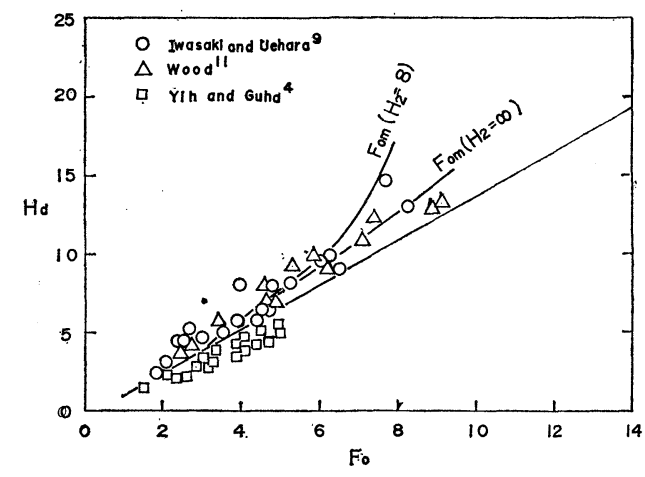

Fig. 6 Comparison with Experimental Data of Other Sources.
Fig. 6 shows plotting of experimental data on conjugate depth of different sources. These data all relate to the internal hydraulic jump of the subsurface flow. Enough information is not provided in these sources as to enable close comparison with the theoretical prediction developed in this paper. However, following remarks can be observed. First, all data except those of Yih and Guha fall above the curve representing the non-mixing internal hydraulic jump. This is in agreement with theoretical predictions of Fig. 3 . Even the data of Yih and Guha may not contradict with Fig. 3 which suggest that some data with small $F_{0}$ may be found below $Q=1$ curve. Secondly these data are scattered around the curves giving maximum $H_{d}$ values for a given $F_{0}$ value, i.e. $F_{0 m}$ curves or envelopes of curves in Fig. 3. In view of this discussion the meaning and use of Figs. 2 and 3 are summarized herein. At first it should be noted again that for given values of $F_{0}$ and $\mathrm{H}_{2}$ these two figures give only the relationship between $Q$ and $H_{a}$. An additional knowledge on the mechanism of mixing is necessary to make the problem physically determinate. In Fig. 2, as the preceding discussion indicates, only the lower segments of the curves $H_{d}=$ constant are physically meaningful. In Fig. 3 for a given set of values of $F_{0}$ and $H_{d}$, the latter to be found only above the line $F_{1}=1$, two values of $Q$ will be found and the smaller of them will actually be realized. For a given set of values of $F_{0}$ and $Q$, the higher of two values of $H_{d}$ obtained in Fig. 3 will be observed.

In practice the densimetric Froude number $F_{0}$ and $H_{2}$ are the only prescribed parameters. Fig. 3 then gives such a useful information as a range of $H_{d}$ values and upper bound of $Q$ value for a given $F_{0}$.

\section{CONCLUSIONS}

Two-dimensional, rapidly varied flow of two layers has been analyzed with use of the impulsemomentum equations. It has been shown that the conjugate depth relationship for both surface and subsurface flow merges under the condition of small density difference. The solution to this problem has been compared with experimental data and it is found that the rate of entrainment induced by the rapidly varied-internal flow is very sensitive to changes in both the upstream and the downstream conditions and that buoyancy reduces the total entrainment to a substabtially smaller amount than would be found either under neutrally buoyant condition or with three-dimen- 
tional buoyant jet flow. The conjugate depth is found to be slightly increased by entrainment of flow. Comparison with experimental data shows that theory predicts the tendency remarkably well.

\section{ACKNOWLEDGEMENTS}

Major part of this work has been carried out while the author was associated with Construction Technique Institute, Foundation, Tokyo, Japan. The author is grateful to their support.

\section{REFERENCES}

1) Koh, R.C. Y.: “Two-Dimensional Surface Warm Jets", J. of Hyd. Div., Proc. ASCE, Vol. 97, HY 6, 1971, pp. 819.

2) Stefan, H.: "Stratification of Flow from Channel into Deep Lake", J. of Hyd. Div., Proc. ASCE, Vol. 96, HY 7, 1970.

3) Schijf, J. B. and Schönfeld, J. C.: "Theoretical Considerations on the Motion of Salt and Fresh Water", Proc. Minnesota International Hydraulic Conv., IAHR, 1953.

4) Yih, C.S. and Guha, C. R.: "Hydraulic Jump in a Fluid System of Two Layers", Tellus, Vol. 7, p. 358.

5) Shi-ighai, H.: "Some Aspects and Experiments on the Density Currents", Trans. JSCE, No. 83, 1962.

6) Hamada, T.: “On Internal Hydraulic Jump”, Dai 5 kai Kaigan Kogaku Koenkai (Fifth Conference of Coastal Engineering), 1958.

7) Iwasaki, T.: "Mitsudo-ryu no Shomondai (Problems of Stratified Flow)", SuikogakuSeries 66-15, JSCE 1966.
8) Hayakawa, N.: “Internal Hydraulic Jump in Co-Current Stratified Flow", J. of Eng. Mech. Div., Proc. ASCE, Vol. 96, No. EM 5, 1970, pp. 797.

9) Iwasaki, T. and Abe, T.: “Intaanaru Jump no Hendo Spectrum ni kansuru Kenkyu (Reseaches on Density Spectrum of Fluctuating Quantities of Internal Hydraulic Jump)", Proc. Dai 18 kai Kaigan Kogaku Koenkai (18th Conf. of Coastal Engineering), 1971.

10) Iwasaki, T. and Abe, T.: "Turbulence in the Weakly Mixing Zone of Interface", International Symposium of Stratified Flows, IAHR, Novosibirsk 1972.

11) Wood, I. R.: “Horizontal Two-Dimensional Density Current", J. of Hyd. Div., Proc. ASCE, Vol. 93, HY 2, 1966, pp. 5139.

12) Wilkinson, D. L. and Wood, I. R.: "A Rapidly Varied Flow Phenomenon in a Two Layered Flow", J. of Fluid Mech., Vol. 47, 1971 , p. 241.

13) Stefan, H. and Hayakawa, N.: "Mixing Induced by an Internal Hydraulic Jump", Water Resources Bulletin, Vol. 8, No. 3, 1972, pp. 531 .

14) Hayakawa, N. and Stefan, H.: “Kongo o Tomonau Naibu Jump ni Tsuite (Internal Hydraulic Jump with Mixing)", Proc. Dai 17 kai Suiri Koenkai (17th Hydraulics Conference), JSCE, 1973.

15) Stolzenbach, K. D. and Harleman, D. R. F.: "Three-Dimensional Heated Surface Jet", Water Resources Research, Vol. 9, No. 1, 1973.

(Received June 5, 1974) 